\title{
Aestuariibacter litoralis sp. nov., isolated from a sandy sediment of the Sea of Japan
}

Correspondence

Naoto Tanaka

n3tanaka@nodai.ac.jp

\author{
Naoto Tanaka, ${ }^{1}$ Lyudmila A. Romanenko, ${ }^{2}$ Galina M. Frolova ${ }^{2}$ \\ and Valery V. Mikhailov ${ }^{2}$ \\ ${ }^{1}$ NODAl Culture Collection Center, Tokyo University of Agriculture, 1-1-1 Sakuragaoka, Setagaya- \\ ku, Tokyo 156-8502, Japan \\ ${ }^{2}$ Pacific Institute of Bioorganic Chemistry, Far-Eastern Branch, Russian Academy of Sciences, \\ 690022 Vladivostok, Prospect 100 Let Vladivostoku 159, Russia
}

\begin{abstract}
The phenotypic and phylogenetic characteristics of an aerobic, Gram-negative, motile, nonpigmented Alteromonas-like bacterium (designated strain KMM $3894^{\top}$ ), isolated from a sandy sediment sample collected offshore of the Sea of Japan, were investigated. Comparative $16 \mathrm{~S}$ rRNA gene sequence analysis revealed that strain $\mathrm{KMM} 3894^{\top}$ belonged to the genus Aestuariibacter and was most closely related to Aestuariibacter halophilus $\mathrm{JC}^{2043}{ }^{\top}$ (95.5\% sequence similarity). Fatty acid analysis showed $\mathrm{C}_{16: 1} \omega 7 c, \mathrm{C}_{18: 1} \omega 7 c$, and $\mathrm{C}_{16: 0}$ as the dominant components. Strain KMM $3894^{\top}$ could be differentiated from recognized species of the genus Aestuariibacter by its ability to grow at $4{ }^{\circ} \mathrm{C}$ and at $30^{\circ} \mathrm{C}$, the optimum temperature for growth, and its inability to utilize most carbohydrates. On the basis of the phenotypic, chemotaxonomic and phylogenetic data, strain $\mathrm{KMM} 3894^{\top}$ is considered to represent a novel species of the genus Aestuariibacter, for which the name Aestuariibacter litoralis sp. nov. is proposed. The type strain is $\mathrm{KMM} 3894^{\top}\left(=\mathrm{NRIC} 0754^{\top}=\mathrm{JCM} 15896^{\top}\right)$.
\end{abstract}

The genus Aestuariibacter was described by Yi et al. (2004) and comprises two species, Aestuariibacter halophilus $\mathrm{JC} 2043^{\mathrm{T}}$ and Aestuariibacter salexigens $\mathrm{JC} 2042^{\mathrm{T}}$, which were isolated from a getbol sediment sample collected from Ganghwa Island, Korea. Here we report the phenotypic and phylogenetic characterization of a Gram-negative, aerobic bacterium, designated strain KMM $3894^{\mathrm{T}}$, which was isolated from a sandy sediment sample collected offshore of the Sea of Japan. Phylogenetic analysis based on 16S rRNA gene sequences indicated that strain KMM $3894^{\mathrm{T}}$ was potentially a novel member of the genus Aestuariibacter. Differential phenotypic properties, together with the phylogenetic distinctiveness demonstrated that strain KMM $3894^{\mathrm{T}}$ differed from other recognized Aestuariibacter species. On the basis of the phenotypic and molecular data obtained, a novel Aestuariibacter species is described.

Strain KMM $3894^{\mathrm{T}}$ was isolated from a sandy sediment sample collected offshore of the Sea of Japan $\left(42^{\circ} 44.348^{\prime} \mathrm{N}\right.$

Abbreviations: ASW, artificial seawater base; MP, maximum-parsimony; $\mathrm{NJ}$, neighbour-joining; SWM, seawater medium.

The GenBank/EMBL/DDBJ accession number for the 16S rRNA gene sequence of strain KMM $3894^{\top}$ is AB473549.

A maximum-parsimony phylogenetic tree based on 16S rRNA gene sequences showing the position of strain $\mathrm{KMM} 3894^{\top}$ and related species is available as supplementary material with the online version of this paper. $\left.133^{\circ} 14.490^{\prime} \mathrm{E}\right)$ at a depth $3 \mathrm{~m}$, as described previously (Romanenko et al., 2004). Strain KMM $3894^{\mathrm{T}}$ was grown aerobically on/in marine 2216 agar (MA; Difco) and marine broth (MB; Difco) or seawater medium (SWM). The SWM medium contained: $5.0 \mathrm{~g}$ peptone, $2.5 \mathrm{~g}$ yeast extract, $1.0 \mathrm{~g}$ glucose, $0.2 \mathrm{~g} \mathrm{~K}_{2} \mathrm{HPO}_{4}, 0.05 \mathrm{~g} \mathrm{MgSO}_{4}$, and $15.0 \mathrm{~g}$ agar, $750 \mathrm{ml}$ natural seawater $/ 250 \mathrm{ml}$ distilled water. The sodium ion requirement and tolerance of various $\mathrm{NaCl}$ concentrations $(0-20 \%)$ were examined on SWM prepared with an artificial seawater base (ASW) (Lyman \& Fleming, 1940), supplemented with an appropriate amount of $\mathrm{NaCl}$. Sea salts requirement was tested using various concentrations of ASW, supplemented with $10.0 \mathrm{~g}$ Bacto peptone $\mathrm{l}^{-1}, 2.0 \mathrm{~g}$ yeast extract $\mathrm{l}^{-1}, 0.028 \mathrm{~g} \mathrm{FeSO}_{4} \mathrm{l}^{-1}$ and $15.0 \mathrm{~g}$ agar $1^{-1}$. Strain KMM $3894^{\mathrm{T}}$ was stored at $-80{ }^{\circ} \mathrm{C}$ in liquid MB supplemented with $30 \%(\mathrm{v} / \mathrm{v})$ glycerol. Motility was observed by using the hanging drop method as described by Gerhardt et al. (1994). Phenotypic properties were tested according to the standard methods described by Smibert \& Krieg (1994). The oxidation/fermentation medium of Leifson (1963) for marine bacteria was used to test acid production from carbohydrates, with $1 \%(\mathrm{w} / \mathrm{v})$ of each compound. Growth at various temperatures and $\mathrm{pH}$ values and in the presence of various $\mathrm{NaCl}$ concentrations and antibiotic resistance were studied as described previously (Romanenko et al., 2003, 2004). In addition, biochemical tests were carried out using API 20NE, API $20 \mathrm{E}$ and API $50 \mathrm{CH}$ test kits (bioMérieux), according to the 
manufacturer's instructions, except that the culture was suspended in ASW. For comparative fatty acid analysis, strain KMM $3894^{\mathrm{T}}$ was cultivated on MA at $28{ }^{\circ} \mathrm{C}$ for 3 days, and lipids were extracted using the chloroformmethanol extraction method of Bligh \& Dyer (1959). Fatty acid methyl esters (FAMEs) were obtained by using alkaline methanolysis ( $15 \% \mathrm{NaOH} /$ methanol). The resultant FAMEs were extracted with hexane and analysed using a GLC-MS Hewlett Packard model 6890 gas chromatograph equipped with a HP 5 MS $5 \%$ phenyl methyl siloxane capillary column $(30 \mathrm{~m} \times 250 \mu \mathrm{m} \times 0.25 \mu \mathrm{m})$ and connected to a Hewlett Packard model 5973 mass spectrometer. A $16 \mathrm{~S}$ rRNA gene sequence of 1504 nucleotides was determined for strain $\mathrm{KMM} 3894^{\mathrm{T}}$, as described by Shida et al. (1997). The sequence obtained was compared with 16S rRNA gene sequences retrieved from the GenBank/EMBL/DDBJ databases by using the FASTA program (Pearson \& Lipman, 1988). Phylogenetic analysis of $16 \mathrm{~S}$ rRNA gene sequences was performed using the software package MEGA 4 (Tamura et al., 2007) after multiple alignment of data by using CLUSTAL_x (version 1.83; Thompson et al., 1997). Phylogenetic trees were constructed by using the neighbour-joining (NJ) and maximum-parsimony (MP) methods and the distances were calculated according to the Kimura two-parameter model. The robustness of the phylogenetic trees was estimated by using a bootstrap analysis of 1000 replicates.
Phylogenetic trees constructed using the NJ and MP methods based on the 16S rRNA gene sequence both gave similar results, as shown, respectively, in Fig. 1 and Supplementary Fig. S1 (available in IJSEM Online). FASTA searches displayed that strain KMM $3894^{\mathrm{T}}$ belonged to the genus Aestuariibacter; Aestuariibacter halophilus JC2043 ${ }^{\mathrm{T}}$ and Aestuariibacter salexigens $\mathrm{JC} 2042^{\mathrm{T}}$ were most closely related to the novel isolate, with 95.5 and $94.9 \%$ sequence similarities, respectively. Strain KMM $3894^{\mathrm{T}}$ shared lower sequence similarities with other species, including Alteromonas genovensis LMG $24078^{\mathrm{T}} \quad(94.4 \%)$, Alteromonas hispanica $\mathrm{F}-32^{\mathrm{T}}$ (94.4\%), Alteromonas stellipolaris LMG $21861^{\mathrm{T}}(93.9 \%)$ and Glaciecola chathamensis S18K6 ${ }^{\mathrm{T}}$ (93.8\%). The 16S rRNA gene sequence similarities obtained for strain KMM $3894^{\mathrm{T}}$ were significantly lower than the threshold similarity value of $97 \%$ proposed by Stackebrandt \& Goebel (1994) and re-evaluated as $98.7 \%$ by Stackebrandt \& Ebers (2006), indicating that strain KMM $3894^{\mathrm{T}}$ could be assigned to the genus Aestuariibacter as an individual species. Strain KMM $3894^{\mathrm{T}}$ contained $\mathrm{C}_{16: 1} \omega 7 c, \mathrm{C}_{16: 0}$ and $\mathrm{C}_{18: 1} \omega 7 c$ as the dominant fatty acids (Table 1), which is in accordance with data reported previously for Aestuariibacter species (Yi et al., 2004). The detailed fatty acid composition of strain KMM $3894^{\mathrm{T}}$ is given in Table 1. The differential phenotypic features of strain KMM $3894^{\mathrm{T}}$ and related species of the genus Aestuariibacter are listed in Table 2 and in the species

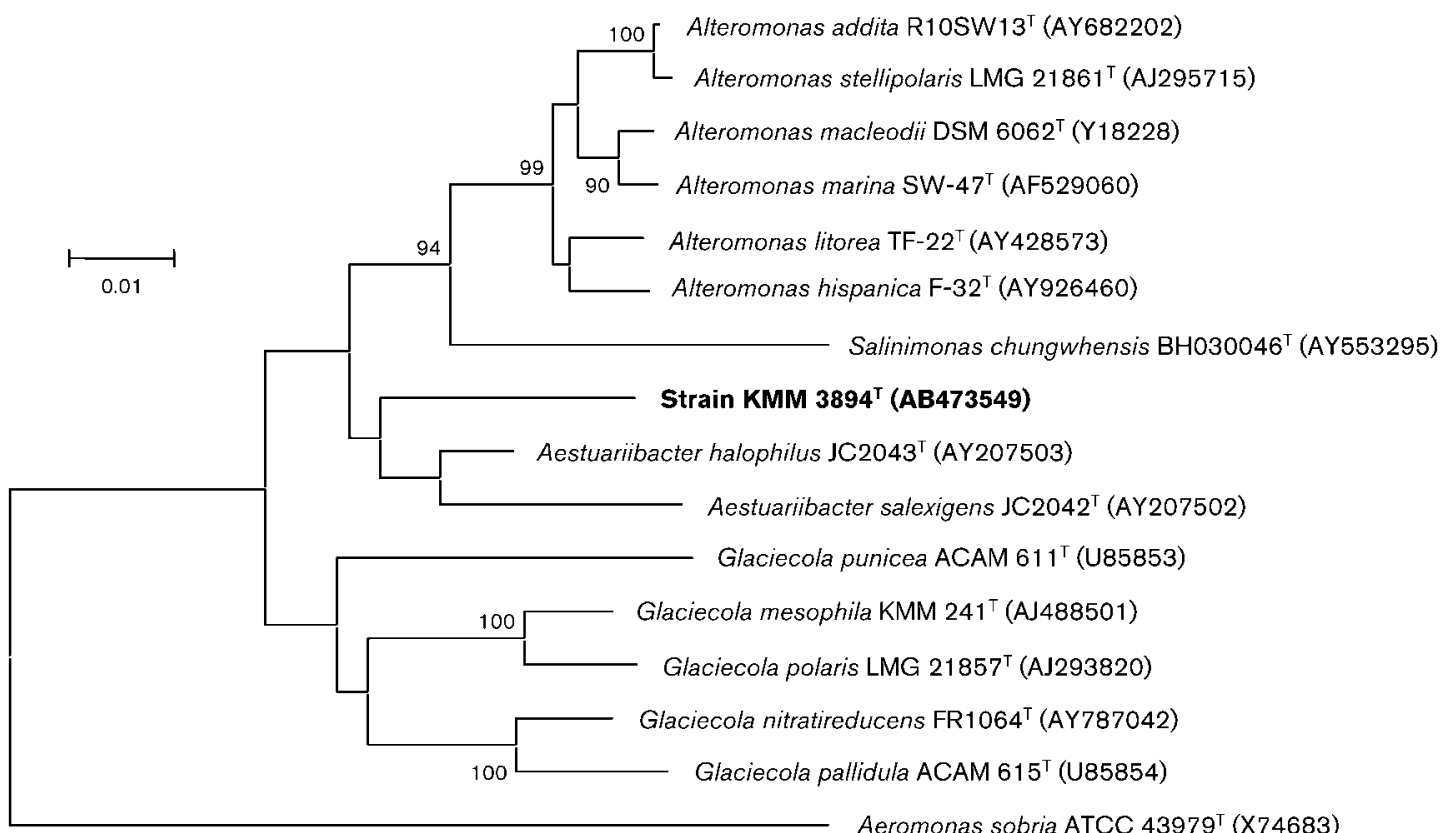

Fig. 1. Neighbour-joining phylogenetic tree based on $16 \mathrm{~S}$ rRNA gene sequences available from the GenBank/EMBL/DDBJ databases (accession numbers are given in parentheses) showing the relationship of strain $\mathrm{KMM} 3894^{\top}$ and recognized Aestuariibacter species. Phylogenetic analysis was performed using the software package MEGA 4 (Tamura et al., 2007) after multiple alignments of data by using CLUSTAL_x (version 1.83; Thompson et al., 1997). Bootstrap values based on 1000 replications are given as percentages at branch points; only values $>90 \%$ are given. Bar, 0.01 substitutions per nucleotide position. 
Table 1. Cellular fatty acid compositions (\%) of strain KMM $3894^{\top}$ and related Aestuariibacter species

Strains: 1, KMM $3894^{\mathrm{T}}$ (Aestuariibacter litoralis sp. nov.); 2, Aestuariibacter salexigens $\mathrm{JC}^{2042^{\mathrm{T}}}$ (data from Yi et al., 2004); 3, Aestuariibacter halophilus $\mathrm{JC} 2043^{\mathrm{T}}$ (Yi et al., 2004). -, Not detected.

\begin{tabular}{|c|c|c|c|}
\hline Fatty acid & 1 & 2 & 3 \\
\hline $\mathrm{C}_{10: 0} 3-\mathrm{OH}$ & - & 0.6 & 4.6 \\
\hline $\mathrm{C}_{12: 0}$ & 2.3 & 1.8 & 3.0 \\
\hline $\mathrm{C}_{11: 0} 3-\mathrm{OH}$ & - & 1.0 & 0.3 \\
\hline $\mathrm{C}_{12: 1} 3-\mathrm{OH}$ & 1.3 & 2.2 & 1.1 \\
\hline $\mathrm{C}_{12: 0} 3-\mathrm{OH}$ & 1.6 & 1.0 & 0.6 \\
\hline $\mathrm{C}_{14: 0}$ & 3.8 & 3.9 & 3.1 \\
\hline $\mathrm{C}_{15: 1} \omega 8 c$ & - & 1.0 & 0.5 \\
\hline $\mathrm{C}_{15: 0}$ & 1.2 & 3.8 & 2.4 \\
\hline $\mathrm{C}_{16: 1} \omega 7 c$ alcohol & - & 2.1 & 0.7 \\
\hline $\mathrm{C}_{12: 0}$ alde/iso- $\mathrm{C}_{16: 1} \mathrm{I} / \mathrm{C}_{14: 0} 3-\mathrm{OH}$ & - & 2.0 & 1.6 \\
\hline iso- $\mathrm{C}_{16: 0}$ & - & 1.1 & 0.1 \\
\hline $\mathrm{C}_{16: 1} \omega 9 c$ & 2.0 & - & - \\
\hline $\mathrm{C}_{16: 1} \omega 7 c /$ iso- $\mathrm{C}_{15: 0} 2-\mathrm{OH}$ & & 27.2 & 32.5 \\
\hline $\mathrm{C}_{16: 1} \omega 7 c$ & 29.1 & & \\
\hline $\mathrm{C}_{16: 0}$ & 17.7 & 15.8 & 23.9 \\
\hline iso- $\mathrm{C}_{17: 1} \omega 5 c$ & - & - & 1.6 \\
\hline $\mathrm{C}_{17: 1} \omega 8 c$ & - & 6.8 & 3.1 \\
\hline $\mathrm{C}_{17: 1} \omega 6 c$ & 3.4 & - & - \\
\hline $\mathrm{C}_{17: 0}$ & 2.7 & 4.5 & 2.8 \\
\hline $\mathrm{C}_{18: 1} \omega 7 c$ & 20.8 & 14.0 & 11.1 \\
\hline $\mathrm{C}_{18: 0}$ & 1.1 & 2.9 & 0.9 \\
\hline $\mathrm{C}_{19: 1} \omega 6 c / \mathrm{C}_{19: 0} \omega 10 c$ cyclo/ $\mathrm{C}_{19: 0} \omega 6 c$ & - & 1.1 & 0.3 \\
\hline
\end{tabular}

description. Strain KMM $3894^{\mathrm{T}}$ could be distinguished from recognized Aestuariibacter species in not being able to grow in artificial sea salts-based medium containing $<20 \%$ sea salts and the ability to utilize most compounds included in the API 20NE, API 20E and API $50 \mathrm{CH}$ panels and in the optimum temperature for growth. Based on the results obtained it is proposed to assign strain KMM $3894^{\mathrm{T}}$ to the genus Aestuariibacter as representing a novel species, Aestuariibacter litoralis sp. nov.

\section{Description of Aestuariibacter litoralis sp. nov.}

Aestuariibacter litoralis (li.to.ra'lis. L. masc. adj. litoralis of the seashore).

Aerobic, Gram-negative, oxidase- and catalase-positive, motile, rod-shaped bacterium (approx. $2 \mu \mathrm{m}$ in length). Colonies are non-pigmented, opaque, whitish and shiny, with wrinkled surface and regular edges of $2-3 \mathrm{~mm}$ in diameter on MA. Growth occurs at $4-40{ }^{\circ} \mathrm{C}$, with optimum growth at $30{ }^{\circ} \mathrm{C}$. Sodium ions are essential for growth. Growth occurs in medium containing 0.5-6\% $\mathrm{NaCl}$ without other sea salts added. No growth is observed on media containing $0.5-12 \%(\mathrm{w} / \mathrm{v})$ artificial sea salts, but is observed on media containing $20-100 \%(\mathrm{w} / \mathrm{v})$ artificial sea salts. $\mathrm{pH}$ range for growth is 5.5-10.5, with optimum growth at $\mathrm{pH}$ 6.5-8.0. Positive for Tween 80, starch, aesculin and DNA hydrolysis. Negative for casein and
Table 2. Differential phenotypic characteristics of strain KMM $3894^{\top}$ and recognized Aestuariibacter species

Strains: 1, KMM $3894^{\mathrm{T}}$ (Aestuariibacter litoralis sp. nov.; data from this study); 2, Aestuariibacter salexigens $\mathrm{JC}^{2042^{\mathrm{T}}}$ (Yi et al., 2004); 3 , Aestuariibacter halophilus $\mathrm{JC}^{2} 243^{\mathrm{T}}$ (Yi et al., 2004). All strains are positive for motility, nitrate reduction and hydrolysis of aesculin, starch, DNA and Tween 80 . All strains are negative for $\beta$-galactosidase and hydrolysis of chitin, agar and cellulose. - , Negative; + , positive; NG, no growth; S, slow reaction; w, weakly positive.

\begin{tabular}{|lccc|}
\hline Characteristic & $\mathbf{1}$ & $\mathbf{2}$ & $\mathbf{3}$ \\
\hline Temperature range for growth $\left({ }^{\circ} \mathrm{C}\right)$ & $4-40$ & $20-40$ & $15-40$ \\
Temperature optimum $\left({ }^{\circ} \mathrm{C}\right)$ & 30 & 35 & 40 \\
Sea salts range for growth $(\%)$ & $20-100$ & $1-10$ & $0.5-10$ \\
Growth in NaCl $(\%)^{*}$ & $0.5-6$ & NG & $1-8$ \\
Hydrolysis of: & & & \\
Casein & - & - & + \\
Gelatin & $\mathrm{S}$ & + & + \\
Utilization of: & & & \\
D-Glucose & - & - & + \\
N-Acetylglucosamine & - & + & + \\
Sucrose & - & - & + \\
Acetate & - & + & + \\
D-Ribose & - & W & + \\
D-Sorbitol & - & W & - \\
& & & \\
\hline
\end{tabular}

${ }^{*}$ Growth in medium containing $\mathrm{NaCl}$, without the addition of sea salts.

chitin hydrolysis, and for $\mathrm{H}_{2} \mathrm{~S}$ production. Gelatin is slowly hydrolysed over $4-5$ days routinely and in API 20NE, and 2 days in API 20E tests. Does not produce melanin-like pigments or clearance zone on medium containing Ltyrosine. Acid is not produced from D-glucose, D-mannitol, sucrose, D-lactose, maltose, D-galactose, D-mannose, cellobiose, D-xylose, D-sorbitol, L-arabinose or L-rhamnose. In addition, according to API 20NE tests, positive for aesculin hydrolysis and nitrate reduction, and negative for indole production, glucose acidification, arginine dihydrolase, urease production and the $p$-nitrophenyl $\alpha$-D-glucopyranoside test, and assimilation of D-glucose, D-mannitol, maltose, L-arabinose, D-mannose, $\mathrm{N}$-acetylglucosamine, D-gluconate, caprate, adipate, L-malate, citrate and phenylacetate. In API 20E tests, positive for trisodium citrate utilization, and negative for ONPG, arginine dihydrolase, lysine decarboxylase, ornithine decarboxylase, $\mathrm{H}_{2} \mathrm{~S}$ production, urease, tryptophan deaminase, indole production, acetoin production, and utilization of D-glucose, Dmannitol, inositol, D-sorbitol, L-rhamnose, sucrose, melibiose, amygdalin and D-arabinose. In API $50 \mathrm{CH}$ tests, weakly positive for utilization of potassium 5-ketogluconate and negative for utilization of glycerol, erythritol, Darabinose, L-arabinose, D-ribose, D-xylose, L-xylose, Dadonitol, methyl $\beta$-D-xylopyranoside, D-galactose, D-glucose, D-fructose, D-mannose, L-sorbose, L-rhamnose, dulcitol, inositol, D-mannitol, D-sorbitol, methyl $\alpha$-D- 
mannopyranoside, methyl $\alpha$-D-glucopyranoside, $N$-acetylglucosamine, amygdalin, arbutin, aesculin, salicin, cellobiose, maltose, D-lactose, melibiose, sucrose, trehalose, inulin, melezitose, raffinose, starch, glycogen, xylitol, gentiobiose, turanose, D-lyxose, D-tagatose, D-fucose, L-fucose, L-arabitol, D-arabitol, potassium 2-ketogluconate and gluconate. Predominant fatty acids are $\mathrm{C}_{18: 1} \omega 7 c, \mathrm{C}_{16: 0}$ and $\mathrm{C}_{16: 1} \omega 7 c$ (detailed fatty acid composition of the type strain is given in Table 2). Susceptible to the following antibiotics (content per disc): ampicillin $(10 \mu \mathrm{g})$, vancomycin $(30 \mu \mathrm{g})$, gentamicin $(10 \mu \mathrm{g})$, kanamycin $(30 \mu \mathrm{g})$, carbenicillin $(100 \mu \mathrm{g})$, chloramphenicol $(30 \mu \mathrm{g})$, nalidixic acid $(30 \mu \mathrm{g})$, oleandomycin $(15 \mu \mathrm{g})$, ofloxacin $(5 \mu \mathrm{g})$, polymyxin $\mathrm{B}(300 \mathrm{U})$, rifampicin $(5 \mu \mathrm{g})$, streptomycin $(30 \mu \mathrm{g})$, erythromycin $(15 \mu \mathrm{g})$, tetracycline $(30 \mu \mathrm{g})$, cephazolin $(30 \mu \mathrm{g})$ and cephalexin $(30 \mu \mathrm{g})$; and resistant to benzylpenicillin $(10 \mathrm{U})$, lincomycin $(15 \mu \mathrm{g})$, neomycin $(30 \mu \mathrm{g})$ and oxacillin $(10 \mu \mathrm{g})$.

The type strain, KMM $3894^{\mathrm{T}}\left(=\mathrm{NRIC} \quad 0754^{\mathrm{T}}=\mathrm{JCM}\right.$ $\left.15896^{\mathrm{T}}\right)$, was isolated from a marine sandy sample, collected offshore of the Sea of Japan, Russia at a depth of $3 \mathrm{~m}$.

\section{Acknowledgements}

This study was supported by a grant from the Presidium of the FarEastern Branch of Russian Academy of Sciences 'Search of marine heterotrophic bacteria biodiversity', and by a grant from the Presidium of RAS 'Molecular and Cell Biology'.

\section{References}

Bligh, E. G. \& Dyer, W. J. (1959). A rapid method of total lipid extraction and purification. Can J Biochem Physiol 37, 911-917.

Gerhardt, P., Murray, R. G. E., Wood, W. A. \& Krieg, N. R. (editors) (1994). Methods for General and Molecular Bacteriology. Washington, DC: American Society for Microbiology.
Leifson, E. (1963). Determination of carbohydrate metabolism of marine bacteria. J Bacteriol 85, 1183-1184.

Lyman, J. \& Fleming, R. H. (1940). Composition of sea water. J Mar Res 3, 134-146.

Pearson, W. R. \& Lipman, D. J. (1988). Improved tools for biological sequence comparison. Proc Natl Acad Sci U S A 85, 2444-2448.

Romanenko, L. A., Schumann, P., Zhukova, N. V., Rohde, M., Mikhailov, V. V. \& Stackebrandt, E. (2003). Oceanisphaera litoralis gen. nov., sp. nov., a novel halophilic bacterium from marine bottom sediments. Int J Syst Evol Microbiol 53, 1885-1888.

Romanenko, L. A., Schumann, P., Rohde, M., Mikhailov, V. V. \& Stackebrandt, E. (2004). Reinekea marinisedimentorum gen. nov., sp. nov., a novel gammaproteobacterium from marine coastal sediments. Int J Syst Evol Microbiol 54, 669-673.

Shida, O., Takagi, H., Kadowaki, K., Nakamura, L. K. \& Komagata, K. (1997). Transfer of Bacillus alginolyticus, Bacillus chondroitinus, Bacillus curdlanolyticus, Bacillus glucanolyticus, Bacillus kobensis, and Bacillus thiaminolyticus to the genus Paenibacillus and emended description of the genus Paenibacillus. Int J Syst Bacteriol 47, 289-298.

Smibert, R. M. \& Krieg, N. R. (1994). Phenotypic characterization. In Methods for General and Molecular Bacteriology, pp. 607-654. Edited by P. Gerhardt, R. G. E. Murray, W. A. Wood \& N. R. Krieg. Washington, DC: American Society for Microbiology.

Stackebrandt, E. \& Ebers, J. (2006). Taxonomic parameters revisited: tarnished gold standards. Microbiol Today 33, 152-155.

Stackebrandt, E. \& Goebel, B. M. (1994). Taxonomic note: a place for DNA-DNA reassociation and $16 \mathrm{~S}$ rRNA sequence analysis in the present species definition in bacteriology. Int J Syst Bacteriol 44, 846849.

Tamura, K., Dudley, J., Nei, M. \& Kumar, S. (2007). MEGA4: Molecular Evolutionary Genetics Analysis (MEGA) software version 4.0. Mol Biol Evol 24, 1596-1599.

Thompson, J. D., Gibson, T. J., Plewniak, F., Jeanmougin, F. \& Higgins, D. G. (1997). The CLUSTAL_X windows interface: flexible strategies for multiple sequence alignment aided by quality analysis tools. Nucleic Acids Res 25, 4876-4882.

Yi, H., Bae, K. S. \& Chun, J. (2004). Aestuariibacter salexigens gen. nov., sp. nov. and Aestuariibacter halophilus sp. nov., isolated from tidal flat sediment, and emended description of Alteromonas macleodii. Int J Syst Evol Microbiol 54, 571-576. 